\title{
TRAINING ALLIES TO REDUCE VARIANCE IN A NURSING HOME STUDY: A COMMUNICATION INTERVENTION
}

\author{
DONNA L. ALGASE \\ Assistant Professor, University of Michigan, Ann Arbor, MI, USA \\ ELIZABETH R. A. BEATTIE \\ Senior Lecturer, Doctoral Candidate, James Cook University, Townsville/University of Michigan, USA
}

\begin{abstract}
SUMMARY
Communication training was undertaken within a study the prime purpose of which is to determine the influence of light levels on rhythms and patterns of wandering behavior in a nursing home sample of cognitively impaired individuals. The purpose of this training was to control variance in the social environment of an experimental lounge since it is posited that a number of environmental influences may impact wandering. Heron six-category intervention analysis (Heron, 1976) was used to interpret the existing social environment and inform ally training. This article outlines ally training methods, introduces training implementation issues and evaluates training outcomes.
\end{abstract}

KEY WORDS - ageing; dementia; control; variance; nursing home; intervention; communication; wandering; environment; volunteer training

The primary goal of the project within which this intervention occurred was to study the influence of light levels on rhythms and patterns of wandering behavior in a nursing home sample of cognitively impaired individuals. Despite an absence of empirically validated theory on wandering, the findings of several studies point to a probable inverse relationship between wandering and social interaction. Monsour and Robb (1982) found that the premorbid personality of wanderers was significantly more sociable than that of nonwandering nursing home residents with cognitive impairment. Despite this preexisting tendency, wanderers show a more rapid decline in social skills than non-wanderers (Cornbleth, 1977). They also engage in less social interaction and exhibit greater social needs (Synder et al., 1978). These findings suggest that for some nursing home residents wandering may substitute for social interaction as cognitive function declines. An association between wandering and social interaction can also be inferred from an observational study of three nursing home residents with Alzheimer's

Address for correspondence: Dr Donna L. Algase, University of Michigan, School of Nursing, 400 North Ingalls, Ann Arbor, Michigan 48109-0482, USA.

CCC 0885-6230/96/100889-05

(C) 1996 by John Wiley \& Sons, Ltd. disease (Hussain, 1989). Of 93 wandering episodes observed, $59 \%$ terminated within 1 foot of another person or persons, whereas $41 \%$ ended in solitary activities. While a number of factors including light, noise, temperature, physical location and social conditions have been posited as environmental influences on wandering, little is known about the relationship between wandering and any of these potential factors. However, more preliminary data are available concerning light than any other factor.

In setting up an experimental environment in which wandering could be studied, it was critical to implement controls for these potential environmental factors. This study is one of the first to attempt to control elements of the social environment of demented subjects in an experimental setting. The focus of the article is a discussion of the extent to which we succeeded in training allies to control variance, and the training and implementation issues in the experimental environment. The methods and outcomes of ally training are also discussed.

The allies were older adults from the general population who volunteered to participate in the research project. Five criteria for the volunteer profile were used: aged 60 or above, or having the appearance of a person that age or above; Accepted 12 September 1995 
Table 1. Volunteer allies profile $(N=23)$

\begin{tabular}{lcc} 
& Male (2) & Female (21) \\
\hline Source: network & 2 & 13 \\
Source: AA & - & 6 \\
Source: alumni & - & 2 \\
Source: resident & - & 2 \\
Affiliation with & 2 & 7 \\
support group & & 11 \\
Disease in family & 2 & 6 \\
Experience as carer & 1 & 6 \\
Previous volunteer & - & \\
$\quad$ experience & & \\
\hline
\end{tabular}

cognitively intact; physically well and mobile; able to commit to a minimum of 2 consecutive hours/ week for 6 consecutive weeks; and a willingness to work with people who had dementia and/or some knowledge of, or experience relating to, people with dementia. Several traditional recruitment strategies were used, including approaching existing volunteer bodies and canvassing church groups and retirement living complexes. However, these approaches failed to yield volunteers. The two most successful strategies, used in the final stages of recruitment, were (1) networking within the personal social networks of research team members and (2) offering incentives, in the form of assessment and expert opinion of problem behaviors, to relatives and carers of people with Alzheimer's disease via support groups. Despite the known significant barriers to recruitment of older people, a cohort of 23 volunteers was recruited over a 9-month period and all those recruited fulfilled their commitment (see Table 1). Training involved a minimum of 4 hours' video and workbook preparation done individually by the ally at home. The whole training, or parts thereof, could be repeated at will by the ally at any time with the project director. In addition, allies received feedback on their interactive behavior with subjects after each session of data collection.

Heron six-category intervention (HSCl) theory was used as the framework both for interpreting the existing social environment of the subjects and for ally training (Heron, 1976). HSCI is a format for understanding the range of useful and therapeutic verbal interactions available to a helper. Helpers can use the HSCI to act more precisely and with a greater sense of intention when relating to a resident. A skilled user can deftly select categories in response to an individual's needs according to a communication plan for achieving specific goals. HSCI skills training offered strengths in that it emphasized the intent of communication, could be readily mastered by individuals without formal backgrounds in communication skills and provided a formal coding system for the purposes of determining retention and reliability (Heron, 1976).

Communication style in the simulated social environment was informed by a 42 -hour observation study of the interactive behavior of demented nursing home residents. Interactive behavior was observed in a lounge at the study site, a part of the subjects' usual environment. The target population was all people who may be present in a lounge in an institution for the aged. The accessible population was all people who may be present in the lounge in the studied nursing home during the hours 7 am$9 \mathrm{pm}$, Monday-Friday inclusive. Random observations divided the time spectrum into 2-hour slots, each of which was observed three times. The sample was a non-probability convenience sample commencing with the first person who initiated a

Table 2. Heron six-category intervention styles

\begin{tabular}{|c|c|c|}
\hline Category & What the nurse/counselor/carer does & Examples of use in experimental setting \\
\hline \multicolumn{3}{|l|}{ Authoritative } \\
\hline Prescriptive* & Makes suggestions, recommends behavior to the patient/client & 'Please come and sit down on this chair.' \\
\hline Informative & Gives new knowledge or information to the patient/client & 'There are puzzles you can do if you want' \\
\hline Confronting & $\begin{array}{l}\text { Challenges restrictive or repetitive attitudes, beliefs or behaviors } \\
\text { of the patient/client }\end{array}$ & \\
\hline \multicolumn{3}{|c|}{ ( } \\
\hline Cathartic & $\begin{array}{l}\text { Helps the patient/client release tension through tears, trembling, } \\
\text { angry sounds or laughter }\end{array}$ & \\
\hline Catalytic & $\begin{array}{l}\text { Helps draw out information from or encourages self-discovery in } \\
\text { the client/patient }\end{array}$ & \\
\hline Supportive* & Affirms the worth of, is supportjve of the client/patient & 'You're doing very well here today.' \\
\hline
\end{tabular}

* Categories utilized in training. 
conversation during each observation period. That person was observed until the interaction ended or until the completion of the 2-hour time period, whichever occurred first. Subsequent subjects were chosen using this interaction rule and interactions were observed sequentially. A coding system was used to distinguish between different types of subjects in the room, for example residents, employees and visitors. Interactional overtures from the subject or others in the lounge were recorded using pencil and paper and organized into a box grid. The vertical axis differentiated between behavior which was socially acceptable and that which was socially unacceptable. The horizontal axis focused on one component of social acceptability, that of intrusion. A behavior could be rated as non-intrusive, intrusive (subject initiated), intrusive (other initiated) or 'other'. Double coding was used to identify the Heron style of the interaction behavior. Results of the observation study indicated a poverty of interactive behavior between residents and staff with the supportive and prescriptive styles being the two predominant ones used by nonresidents, together accounting for over $90 \%$ of the observed verbal interactions. Residents initiated about $15 \%$ of total interaction on the observation study and their predominant style was prescriptive.

Although HSCI offers six potential interaction styles, the volunteers were trained in only the two predominantly observed styles, supportive and prescriptive, since the purpose of training the allies was not to have them become excellent communicators or therapeutic agents. Rather, it was to have them communicate in consistent styles in the experimental room, across allies and subjects, such that their styles simulated the lounge environment and their level of consistency in communication could be evaluated. The intent of supportive style is to help an individual feel safe, comfortable and valued as a person in an interaction. It is designed for use in all interactions regardless of their overall purpose. Supportive style conveys respect and genuineness and is fundamental to creating a climate in which it is possible to establish rapport with an individual (Heron, 1976). In the center of this study the main purpose for the supportive style was to establish a level of social comfort and trust in order that subjects would readily remain in the experimental room without difficulties. In addition, non-resident initiated social interaction patterns were supportive $31 \%$ of the time in the observational study.

The prescriptive style is used predominantly to direct the behavior of an individual in a specific way. It is designed for use when an individual needs assistance in making decisions or when a particular behavior is desirable in a specific context (Heron, 1976). With cognitively impaired individuals prescriptive communication can serve to distract a person from repetitive, socially irritating or potentially harmful behaviors. In the context of this study, the purpose for the prescriptive style was twofold: first, to enhance the reality of simulated experimental setting, since $58 \%$ and greater than $85 \%$ of the observed interactions of residents and non-residents, respectively, were prescriptive; second, to give allies a specific tool or help them defer interaction with the subject if the subject became preoccupied with their presence in the environment. Coupling Heron supportive and prescriptive styles allowed us a level of control over the emotional tone without simulating an excessively warm or cold interactive environment significantly dissimilar to the everyday.

Some allies who were close to a person with dementia displayed natural non-verbal cueing and warmth levels unusual in individuals with no such relationship, although this observation was not systematically validated. To control for this feature, as well as to simulate the communication in the lounge, allies received instruction in the use of three additional communication skills: minimal response techniques, congruency and limited warmth (Sundeen et al., 1986). Minimal response techniques functioned in two ways. First, they assisted an individual to interact and to disclose thoughts and feelings, especially when accompanied by eye contact and other attending behaviors. Second, they precluded a person interacting or disclosing when accompanied by attending behaviors. Example of a minimal response technique was to indicate to the subject that the ally was occupied with his/her own activity and did not want to communicate right now. This response left the subject free to behave as desired. Congruency refers to allies achieving a match between verbal and non-verbal elements of the communication, ie between what is said and what is done in order that their messages to the subject are clear. Appropriate social distance was achieved by allies limiting physical display of warmth, for example not hugging, holding hands with, or sitting too close to the subject. Coupling minimal response techniques, congruency and limited physical warmth with prescriptive communication allowed us a greater measure of control over the likelihood of allies reverting to 'natural' interactive styles. Since the 
allies were external volunteers, not staff routinely involved in resident care, the communication modification never extended beyond the research situation.

\section{TRAINING TECHNIQUES}

Two training techniques were used to instruct allies: a video and accompanying written reference and review materials for use with the video. Video training was chosen over other forms of instruction because it offered several advantages: realistic situations on record, the illustration of pure forms of the interactive styles, a repeated practice option and ease of access for the ally at a relatively low cost. The choice of scenarios was critical in providing the allies with training that would equip them to deal with most situations they would encounter with a subject. Given that subjects in this study were cognitively impaired, allies had to be aware of common behaviors associated with dementia and how to respond to such behaviors in the experimental setting. However, having spent time and energy recruiting and training allies, we did not want to worry them unduly by presenting the demented as troublesome or difficult. Training was important because in some circumstances the usual or natural response of the ally might be categorically opposed to the desired behavior, for example, a natural inclination to be very friendly and chatty with a subject on the part of the ally was problematic in data collection terms.

It was possible to create realistic guided imagery and interaction vignettes using video, illustrating aspects of both the desirable and less desirable communication skills required of the allies. We used the analogy of an airport lounge in which you are reading a good book and an anxious traveller enters. This scenario was designed to evoke feelings an ally may experience working with a cognitively impaired subject, for example irritation, sadness, anger, embarrassment, sympathy and the wish to help. The ally was given permission to feel deeply about the subject but not to act on the basis of those feelings, but rather as we had trained them to behave. The interactive vignettes illustrated a range of subject behaviors, specifically those which allies might find difficult or confronting or might lead to a data collection session being interrupted or stopped. The video was hand-delivered to allies, facilitating access to training and introducing allies to the team member who was most closely associated with data collection prior to a data collection session.

Two manuals were written for use by allies. A Volunteer Information, Video and Training Manual introduced allies to the research team and the experimental environment. It included fundamental concepts involved in the ally role, for example: the purpose of the study, elements of the research environment (cameras, altered light levels), confidentiality, safety, access to and behavior appropriate to the study site, and what to do before, during and after a data collection session. A large section in this manual focused on preparation for the role of ally, including guidelines for the use of the video and accompanying workbook. The Video Training Workbook was a competency-based workbook for use before, during and after the video. Section A of this workbook reviewed and tested knowledge and understanding of the material contained in the information manual using 10 sentence completion questions. Section $B$ of the workbook introduced the Heron communication style concepts, minimal response techniques, congruency and limited warmth. It tested knowledge and understanding of desirable communication styles in 20 choice questions. Ten questions were based on the recognition of styles and 10 on appropriate responses to written scenarios similar to those presented on the video. The written manual/workbook technique allowed the team to present information in a second format that was more accessible than videotape and accommodated different ally learning styles. The manual was small and light enough to be easily carried for reference and review purposes. Having a hard copy of instructions reinforced for allies the importance of their role and the seriousness with which we viewed their training.

Post-training analysis of the interactive behavior of the allies in data collection sessions is limited to those interactions which could be overheard because the experimental room is not equipped with sound recording facilities. The coding system utilized four categories: HSCI supportive, HSCI prescriptive, MRT and other (non-trained styles). The ally's response only in the interaction was coded, not the subject's speech. It was possible to fully code allies' interactive behaviors occurring in 72 sessions out of a total of 1712 -hour data collection sessions completed. Partial coding of interactive behaviors was possible for another 64 sessions, leaving 35 sessions uncoded. It was not possible to code all sessions as the field coordinator 
in charge of coding was sometimes required to participate when volunteers were absent. For coding purposes an interaction was defined as an exchange involving one verbal utterance initiated by the subject and the ally's response to this utterance. From the 72 fully coded sessions the total number of interactions was 261 , with a mean subject-initiated interaction rate of 3.6 interactions per session and a modal rate of 3 . Allies responded in a supportive style in $68 \%$, prescriptive $27 \%$, MRT $5 \%$ and other $0 \%$ of interactions. From the 64 partially coded sessions the total number of audible reported interactions was 143 , with a mean subject-initiated interaction rate of 2.2 interactions per 2-hour session and a median interactive rate of 2. These interaction rates illustrate a similar pattern of interactive poverty in the initiated social behavior of the subjects to that evidenced in our initial 42-hour observation study.

Analysis of the results of the post-experience questionnaire completed by allies (16) indicated that the overall ally experience had been positive (see Table 3). However, despite this overall positive experience, only $26 \%$ (6) of allies indicated that they would be happy to volunteer for this type of project again. Reasons for this are speculative and may not relate to working with a demented peer or to the length and scheduling of sessions. Further investigation of this is indicated.

In conclusion, allies learned their role very well. They were able to perform consistently in terms of the communication behaviors they had been trained to display. However, a significant level of communication contrast was apparent between the prescribed role of ally and the role of research assistant. The research assistant had the responsibility to ensure that each subject willingly partici-

Table 3. Summary of post-experience ally questionnaire results

\begin{tabular}{lcc}
\hline Aspect of experience & Yes & No \\
\hline $\begin{array}{l}\text { Felt welcomed and respected by } \\
\text { research team }\end{array}$ & $100 \%$ & - \\
$\begin{array}{l}\text { Felt well prepared for the ally role } \\
\text { Felt training was clear, realistic }\end{array}$ & $100 \%$ & - \\
$\quad$ and appropriate & $82 \%$ & $18 \%$ \\
$\begin{array}{l}\text { Were worried or upset by aspect/s } \\
\text { of subjects' behavior }\end{array}$ & $20 \%$ & $80 \%$ \\
$\begin{array}{l}\text { Learned something about dementia } \\
\text { from the experience }\end{array}$ & $100 \%$ & - \\
$\begin{array}{l}\text { Would volunteer for this type of } \\
\text { project again }\end{array}$ & $26 \%$ & $74 \%$ \\
\hline
\end{tabular}

pated in data collection on each scheduled occasion. High participatory rates were achieved by the assistant's use of a warm and encouraging interpersonal style and high levels of Heron supportive and informative behavior. Allies were brought into the experimental room first and then the subject was brought in after the ally was settled. The ally watched and listened to the research assistant's behaviors, which were designed to get the subject into the room and to remain there initially. The assistant's warm style directly conflicted with the MRT and reduced the social warmth the allies were trained to display. In some circumstances allies may have been influenced by unintentional modeling of warmth by the RA to the extent that they became overly warm with the subject. It is possible that this heightened warmth level was extended into and across the data periods of those allies affected by it and is reflected in the high proportion of supportive behaviors observed. Despite this deviation from training and propensity of several allies to initiate interaction with the subject, high levels of adherence to training were achieved. The tendency of some allies to initiate interaction may have reduced wandering behavior in some subjects.

Interestingly, regardless of allies' behavior or lighting conditions, early data analysis from this study indicates that subjects wandered little within the experimental environment. Therefore, it is important to question whether the pattern we are seeing is evidence of the inverse relationship between wandering and social interaction observed by others or a function of other physical factors such as light within the environment.

\section{REFERENCES}

Cornbleth, T. (1977) Effects of a protected hospital ward area on wandering and non-wandering geriatric patients. $J$. Gerontol. 32, 573-577.

Heron, J. (1976) A six-category intervention analysis. Brit. J. Guidance Counselling 4(2), 143-155.

Hussain, R. A. (1982) Stimulus control in the modification of problematic behavior in elderly institutionalized patients. Int. J. Behav. Geriat. 1, 33-42.

Monsour, N. and Robb, S. S. (1982) Wandering behavior in old age: A psychosocial study. Soc. Work 27(5), 411-416.

Snyder, L. H., Rupprecht, P., Pyrek, J., Breckhus, S. and Moss, T. (1978) Wandering. Gerontologist 18(3), 272-280.

Sundeen, Stuart, Rankin and Cohen (1986) Nurse-Client Interaction, 3rd. edn. Rank, Sydney. 